\title{
UTILIZAÇÃO DE TRATAMENTO OXIDATIVO AVANÇADO NA DEGRADAÇÃO DO POLUENTES EMERGENTE PARACETAMOL
}

\author{
L. S. G. ARAÚJO' ${ }^{1}$, J. D. B. COSTA-FILHO ${ }^{1}$, C. K. O. SILVA-RACKOV', M. M. G. R. \\ VIANNA $^{2}$ e O. CHIAVONE-FILHO ${ }^{1}$ \\ ${ }^{1}$ Universidade Federal do Rio Grande do Norte, Departamento de Engenharia Química \\ ${ }^{2}$ Universidade de São Paulo, Departamento de Engenharia Química \\ E-mail para contato: Leysa.guedes@gmail.com
}

\begin{abstract}
RESUMO - Estudos relatam que uma grande variedade de fármacos são encontrados em diversos corpos d'água, como rios e lagos. Este tipo de poluente emergente também caracterizado como micropoluente, têm sido motivo de preocupação e chamam a atenção da comunidade científica, uma vez que as estações de tratamento convencionais de água e efluentes, não possuem eficácia suficiente para removê-los do meio. Atualmente existem algumas alternativas para o tratamento desses, os processos de oxidação avançada (POA) são alternativas novas e eficazes para a degradação desses poluentes emergentes. $\mathrm{O}$ presente trabalho estudou a degradação do fármaco paracetamol em solução aquosa, através de oxidação avançada por persulfato de sódio, ativado por metal e variação de $\mathrm{pH}$. Foram realizados ensaios com efluente simulado em concentração de 100 ppm do fármaco, em sistema de batelada em agitação magnética constante. Foi possível observar de acordo com os resultados obtidos que não ocorreu mineralização do composto, entretanto, a degradação do mesmo é evidente e depende de forma significativa do método de ativação e da concentração do oxidante. Seu maior percentual de degradação foi observado em pH ácido e com menores concentrações de persulfato e maiores de catalisador. Esses resultados são úteis para compreensão da remediação de águas contendo esses contaminantes emergentes por meio de processos oxidativos avançados.
\end{abstract}

\section{INTRODUÇÃO}

Em todo o mundo são consumidas anualmente uma quantidade expressiva de fármacos de diferentes classes. Dentre os mais comuns pode-se citar os antipiréticos, analgésicos, reguladores lipídicos, antibióticos, antidepressivos, agentes quimioterápicos, drogas contraceptivas e outros diversos. Após a utilização, esses compostos são parcialmente metabolizados e excretados na urina e fezes, e, por conseguinte percorrem um caminho comum a maioria dos dejetos humanos, entram nas estações de esgoto (ETEs), onde são tratados, juntamente com outros constituintes orgânicos e inorgânicos do efluente. Porém, estudos mostram que alguns destes fármacos não são completamente removidos nas ETEs (GEBHARDT e SCHRÖDER, 2007; TERNES, 1998). 
O esgoto doméstico tem representado um grande problema para a qualidade e manutenção da vida aquática, mesmo sendo tratados em estações de tratamento (ETE), pois existe uma grande quantidade e variedade de compostos. Com a diversidade de compostos depositados nos esgotos, o grupo de poluentes emergentes tem ganhado maior atenção. (FIELD, ET AL, 2006; BELL, ET AL., 2011; ELLIS, 2006; IKEHATA, ET AL., 2006; MEFFE E BUSTAMANTE, 2014; WELLS, ET AL., 2007; WELLS, ET AL., 2008).

Os poluentes emergentes (PE) vêm sendo foco de preocupação, e estão sendo alvo de estudo mundial por apresentarem potencial ameaça ao ambiente, à saúde humana, por não possuírem valores orientadores e nem métodos analíticos regulamentados pelos órgãos ambientais (DEBLONDE, 2011). Este grupo de contaminantes apresenta mais de 40.000 substâncias que incluem fármacos, produtos de higiene pessoal, hormônios sintéticos e naturais, pesticidas e plastificantes, do qual muito pouco é conhecido sobre seus efeitos na vida aquática e na saúde humana (CETESB, 2013).

Apesar do elevado consumo destes outros potenciais contaminantes, os fármacos estão como os mais consumidos no mundo, com destaque para o Paracetamol, que quando ingerido em doses elevadas pode causar danos ao fígado em humanos e animais (NAPOLEAO et al,

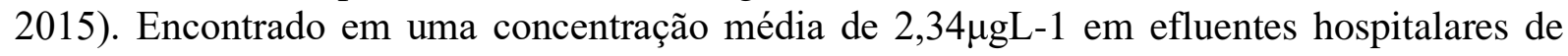
acordo com Verlicchi et al (2002).

O paracetamol (N-(4-hidroxifenil)etanamida - Nome IUPAC) é um fármaco com propriedades analgésicas e antitérmicas largamente utilizado mundialmente, e vem sendo detectado em efluentes de diversos países. Nos últimos anos, o desenvolvimento de tecnologias para a degradação de paracetamol tem recebido grande atenção, por esse poluente ser resistente às tecnologias convencionais aplicadas no tratamento de água.

Embora remoções insuficientes de muitos micropoluentes de água potável por meio de processos convencionais de tratamento tenham sido observados, tecnologias avançadas têm demonstrado grande habilidade para degradar/remover muitos destes micropoluentes. Em particular, os Processos de Oxidação Avançada (POA) são meios eficazes para degradar estes compostos durante tratamento de água potável (IKEHATA et al, 2006; JIN et al, 2012).

A partir dessa perspectiva, este trabalho tem como principal objetivo investigar o tratamento do paracetamol (PCT). Para essa substância foi investigado o tratamento oxidativo, utilizando persulfato de sódio ativado por metal e $\mathrm{pH}$.

\section{METODOLOGIA}

Neste trabalho foi utilizada uma amostra comercial do fármaco para simulação do efluente, com concentração de $750 \mathrm{mg}$ de paracetamol. As soluções foram preparadas em água destilada, obtida por meio da condensação de vapor d'água impura. Os experimentos foram realizados com o fármaco em concentração de $100 \mathrm{mg} / \mathrm{L}$ e volume de $500 \mathrm{~mL}$ de água destilada. Os ensaios ocorreram em reações contínuas com agitação magnética constante, variando os valores de $\mathrm{pH}(3,7$ e 11), catalisador e concentração de oxidante, em uma temperatura média de $25^{\circ} \mathrm{C}$. Onde alíquotas de $8 \mathrm{~mL}$ da amostra foram retiradas de cada erlenmeyer nos tempos $0,15,30,45,60$ e 90 min de reação. 
A quantificação do teor de degradação do analito foi realizada utilizando as técnicas de análise de carbono orgânico total (TOC) e cromatografia líquida de alta eficiência (CLAE). Para as análises de CLAE uma extração foi requerida. A técnica de extração utilizada para esta análise consistiu na extração de fase sólida (solid phase extraction - SPE), utilizando cartuchos SPE C18. Essa técnica foi realizada em 5 etapas como mostrado na Figura 1: cartucho pré- condicionado com $10 \mathrm{~mL}$ de acetonitrila; estabilizado com $5 \mathrm{~mL}$ de água ultrapura; carregado com $3 \mathrm{~mL}$ da amostra; lavado com $5 \mathrm{~mL}$ de água ultrapura; e, finalmente eluído com $1,5 \mathrm{ml}$ de acetonitrila e acondicionado em vial de $1,5 \mathrm{~mL}$ para análise no cromatógrafo.

Figura 1 - Extração em fase sólida (solid phase extraction - SPE).

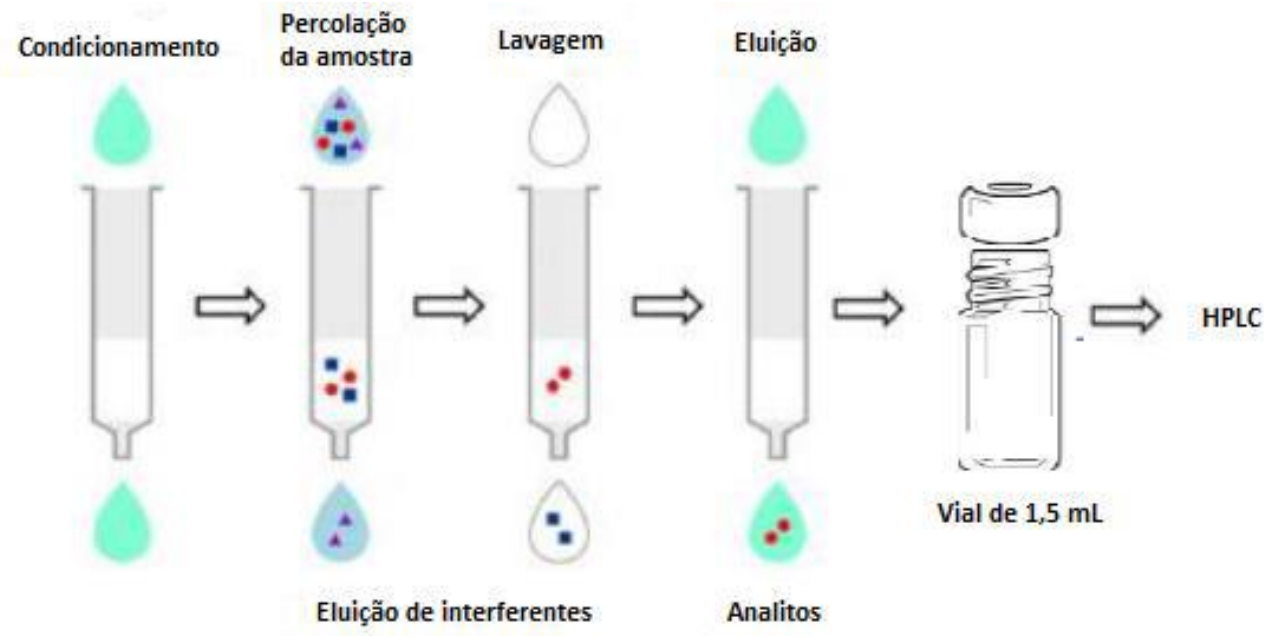

A degradação ocorreu em erlenmeyer com agitação constante formando vórtex na solução, onde foi adicionada a massa de catalisador e persulfato de sódio após o ajuste do $\mathrm{pH}$. O catalisador foi elaborado de acordo com o método descrito por Silva (2014), utilizando diatomita modificada por (Fe2(SO4)3) e FeSO4.7H2O com uma solução de $\mathrm{NaOH}$. A concentração do Paracetamol (PCT) foi quantificada por cromatografia líquida de alta eficiência (HPLC), empregando-se um equipamento Shimadzu (modelo 10 AD) equipado com um detector UV (modelo SPD-20A). O volume de injeção utilizado foi $25 \mu \mathrm{L}$, vazão de $1 \mathrm{~mL} / \mathrm{min}$, coluna C18 Varian $(150 \mathrm{~mm} \times 4,6 \mathrm{~mm}, 5 \mu \mathrm{m})$ e detector UV ajustado para os comprimentos de onda 225 e $254 \mathrm{~nm}$. A concentração de carbono orgânico total (TOC) foi determinada em um equipamento Shimadzu (modelo 5000A).

\section{RESULTADOS E DISCUSSÕES}

Para a avaliação da degradação do fármaco paracetamol utilizou-se a oxidação avançada como técnica, sendo o persulfato de sódio o oxidante responsável pela degradação do composto. É possível observar na Figura 2, que o maior percentual de degradação do poluente ocorreu na mesma faixa de concentração de oxidante e $\mathrm{pH}$, sendo elas, $10 \mathrm{~g} / \mathrm{L} \mathrm{e} \mathrm{pH} 3$, onde alterando a massa de catalisador obtêm-se praticamente o mesmo percentual de degradação, variando entre 95 e $97 \%$. 
Figura 2 - Degradação do PCT em diferentes concentrações de Na2S2O8 e quantidade de catalisador à temperatura constante de $25^{\circ} \mathrm{C}$.

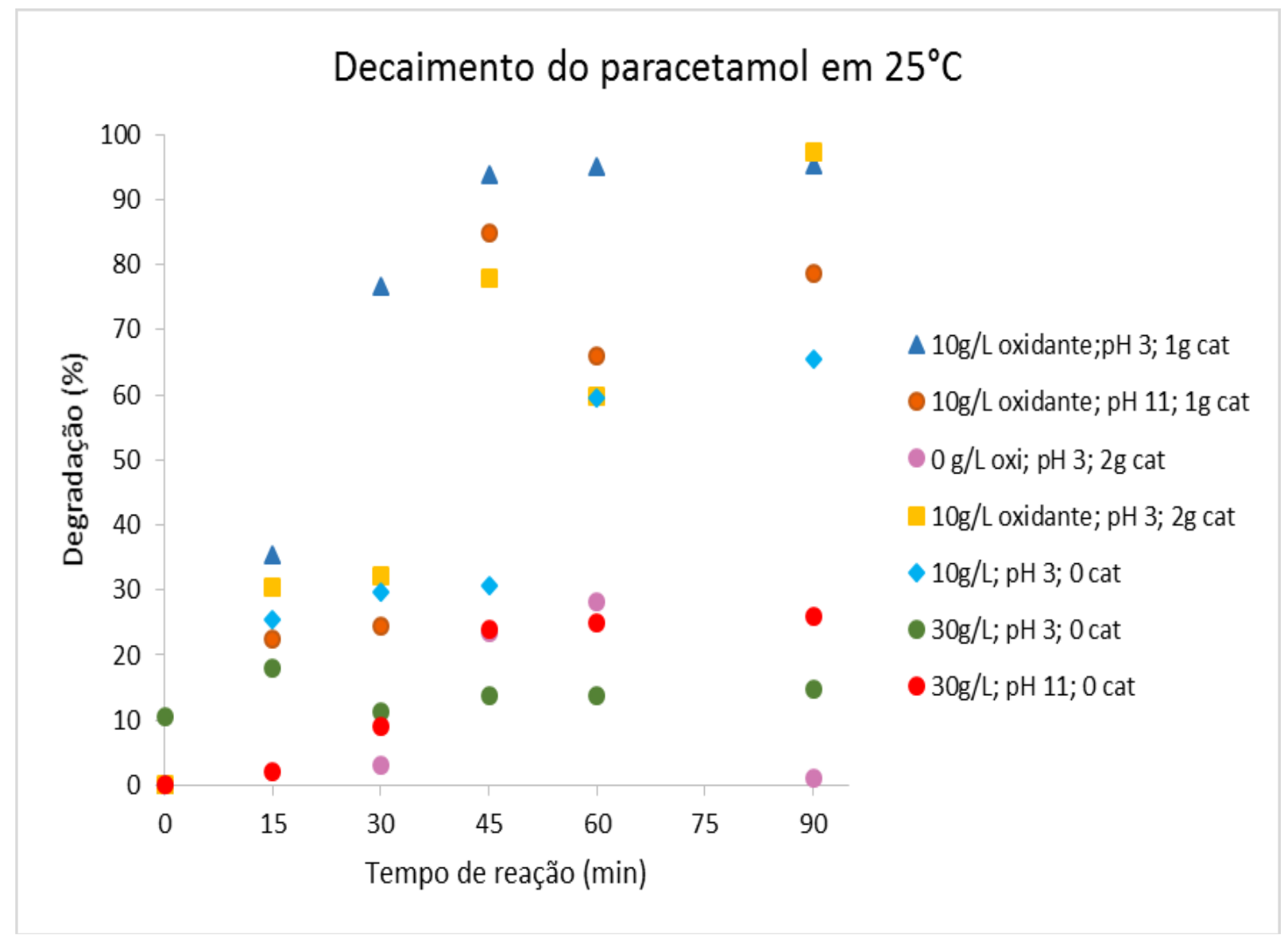

Avaliando os pontos mostrados na Figura 2, pode-se constatar também que o menor percentual de degradação ocorreu com uma alta concentração de oxidante, e uma pequena massa de catalisador, o que permite afirmar que a eficiência do processo não está diretamente ligada ao alto teor de oxidante no meio, o crescimento não é linear. Isto pode ser explicado devido ao excesso de persulfato de sódio nas condições utilizadas, provavelmente está havendo competição no sistema, diminuindo assim a quantidade de radicais sulfato formados, tornando o sistema menos reativo e consequentemente diminuindo a eficiência da degradação. Para esses ensaios considera-se que a variável quantidade de catalisador apresentou maior significância quando utilizado em conjunto com o persulfato de sódio, promovendo assim de forma mais eficiente a ativação do oxidante para degradação do PCT.

No tratante aos resultados obtidos pela análise do teor de carbono orgânico total, é possível observar na Figura 3, que mostra linhas tênues no decorrer do tempo reacional, que o paracetamol não é mineralizado durante a reação, porém sua degradação ocorre, ou seja, compostos intermediários desconhecidos são formados durante o processo. 
Figura 3 - Mineralização do PCT com diferentes concentrações de Persulfato de sódio e quantidade de catalisador à temperatura constante de $25^{\circ} \mathrm{C}$.

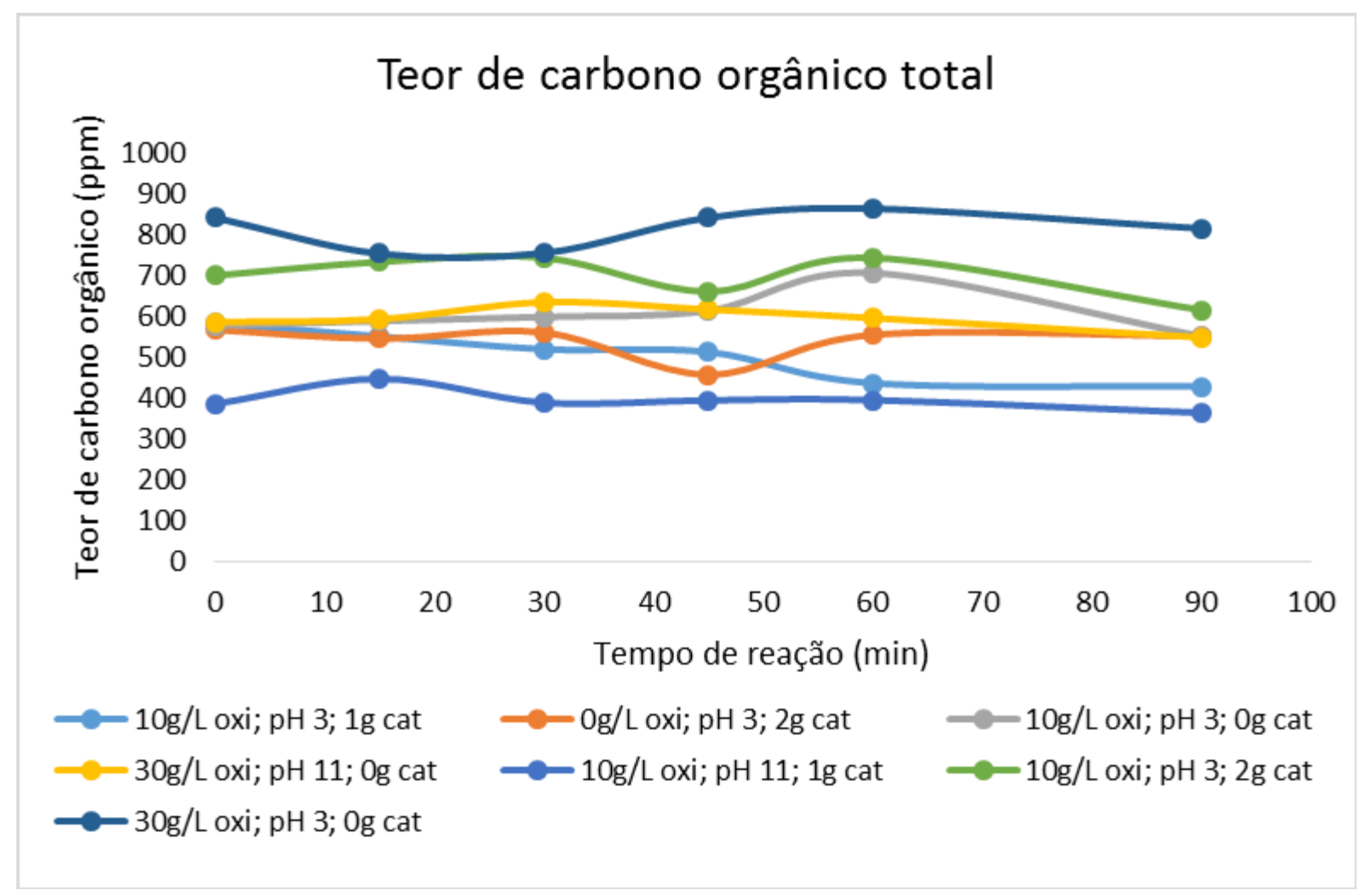

A partir da análise da Figura 3, é possível notar a linearidade dos pontos e, portanto, pouca ou nenhuma mineralização do composto.

\section{CONCLUSÕES}

Os tratamentos oxidativos avançados estão sendo satisfatórios na degradação do poluente emergente paracetamol, uma vez que os percentuais de degradação são bastante elevados, e crescem de acordo com as mudanças de condições para ativação do oxidante. É notável que no tratamento com persulfato de sódio à temperatura constante, sua ativação é favorecida por $\mathrm{pH}$ ácido $(\mathrm{pH}=3)$, baixas concentrações de oxidante e maior concentração de metal, aumentando a quantidade de radicais sulfato. $\mathrm{O}$ melhor percentual de degradação dos casos estudados foi de 97\%, nas condições de baixa concentração de oxidante (10g/L), pH ácido $(\mathrm{pH}=3)$ e pequena quantidade de catalisador $(1 \mathrm{~g})$, enfatizando o que foi discutido anteriormente. Também se conclui que no decorrer do processo praticamente não ocorre mineralização do composto, entretanto, ocorre a degradação do composto formando outros intermediários, que serão investigados em trabalhos futuros.

\section{REFERÊNCIAS}

BEEL, K. Y.; WELLS, M. J. M.; TRAEXLER, K. A.; PELLEGRIN M.; MORSE A.; BANDY, J.; Emerging Pollutants. Water Environment Research, v. 83, p. 1906-1984, 2011. 
CETESB - Companhia de Tecnologia de Saneamento Ambiental. Relatório de áreas contaminadas no Estado de São Paulo, 2013a. Poluentes emergentes. Disponível em: <http://www.cetesb.sp.gov.br/noticia/567,Noticia>. Acesso: 31 ago 2014. DEBLONDE, T.; COSSU-LEGUILLE, C.; HARTEMANNA, P. Emerging pollutants in wastewater: A review of the literature. International Journal of Hygien and Enviromental Health, 214(6), 2011, 442-448.

ELLIS, J. B.; Pharmaceutical and personal care products (PPCPs) in urban receiving waters. Environmental Pollution, v. 144,p. 184-189, 2006.

FIELD, J. A.; JOHNSON, C. A.; ROSE, J. B.; Whats "Emerging"? Environmental Science \& technology, v. 40, p. 7105- 7105, 2006.

GEBHARDT W., SCHRÖDER H.Fr. Liquid chromatography-(tandem) mass spectrometry for the follow-up of the elimination of persistent pharmaceuticals during wastewater treatment applying biological wastewater treatment and advanced oxidation. J. Chromatogr. A, v.1160, p.34-43, 2007.

IKEHATA, K., NAGHASHKAR, N.J., EI-DIN, M.G. Degradation of aqueous pharmaceuticals by ozonation and advanced oxidation processes: A review. Ozone Sci. Eng., 28 (6), 2006, 353- 414.

Napoleão, DC, Silva, RF, Souza, DP, Silva, PTS, Duarte, MMMB, Benachour, M, SILVA, VL. Validação de método analítico de identificação e quantificação dos contaminantes emergentes ácido acetilsalicílico, diclofenaco e paracetamol utilizando análise de LC/MS -IT-TOF. Química no Brasil. 2013; 7: 25-32.

SILVA, C.K.O. Método inovador de ativação de oxidantes no tratamento de poluentes prioritários e emergentes. 2015. 120 f. Tese (Doutorado) - Escola Politécnica da Universidade de São Paulo. Departamento de Engenharia Química, São Paulo. 2014.

TERNES T.A. Occurrence of drugs in german sewage treatment plants and rivers. Water Res., v.32, n.11, p.3245-3260, 1998.

VERLICCHI, P.; AUKIDY, M.A.; GALLETTI, A.; PETROVIC, M.; BARCELÓ, D. Hospital effluent: Investigation of the concentrations and distribution of pharmaceuticals and environmental risk assessment. Science of the Total Environment, 430, 109-118, 2012 